\title{
Núcleo básico en el análisis de datos cualitativos: pasos, técnicas de identificación de temas y formas de presentación de resultados
}

\author{
Basic Core in the Analysis of Qualitative Data: Steps, Techniques to Identify \\ Themes, and Data Display Forms
}

\begin{abstract}
Pablo Ezequiel Flores-Kanter ${ }^{1}$, Leonardo Adrián Medrano²
${ }^{1}$ Licenciado en Psicología; Investigador Part-Time de la Secretaría de Investigación de la Universidad Empresarial Siglo XXI. E-mail: ezequielfk@gmail.com

${ }^{2}$ Doctor en Psicología; Secretario de Investigación de la Universidad Empresarial Siglo XXI.

Universidad Empresarial Siglo XXI. Córdoba, Argentina
\end{abstract}

\begin{abstract}
Resumen
En la investigación cualitativa, el análisis y la interpretación de los datos es una tarea de difícil manejo, incluso para los investigadores más experimentados. Si bien existen muchas técnicas disponibles para llevar a cabo el análisis de datos cualitativos, algunos autores relevantes del área proponen que es posible identificar un núcleo básico sin necesidad de hacer coincidir las distintas perspectivas del método cualitativo. Hacer foco en esta comunalidad permitirá hacer más comprensible la tarea de análisis para los investigadores noveles. El objetivo principal del presente trabajo es mostrar en qué consiste este núcleo básico de análisis, dando cuenta de los pasos necesarios para llevarlo a cabo. Además, se revisan técnicas concretas para la detección de temas, se presentan ejemplos haciendo uso del software Atlas.ti, y se muestran las formas posibles de presentación de los resultados.
\end{abstract}

Palabras clave: Análisis de Datos Cualitativos; Núcleo Básico; Técnicas para Identificar Temas o Categorías; Codificación; Atlas.ti.

\begin{abstract}
Within the research process, the analysis of the data emerges as one of the most important steps. In qualitative research, the analysis of data is a difficult task for even the most experienced researchers and often brings up many doubts about the way to implement it. It is therefore necessary to have material that facilitates the analysis process. Even though there are numerous manuals that focus on the analysis of qualitative data, researchers often can be confused with the large number of names that this type of analysis receives (e.g. Thematic Analysis, Content Analysis) or with the various qualitative methods (e.g. Phenomenology, Grounded Theory) that are available. Each of these qualitative approa-
\end{abstract}


ches presents a particular language to detail the research process, which makes it difficult to recognize common aspects shared by these methods. Recently, the American Psychological Association has emphasized the need to identify, within the various qualitative methods and procedures, shared standards for reporting this type of work. In agreement with the above, several qualitative researchers have pointed out that beyond the aforementioned diversity it is possible to identify a basic core with regard to qualitative analysis, without having to match the different perspectives of the qualitative method, such as Grounded Theory, Ethnography ore Phenomenology. Focusing on this communality will facilitate a simpler and clearer approach to the data analysis process. The analysis process mainly involves 1) data condensation, and 2) presentation of results. Following this line, the present manuscript aims to: (a) develop what the basic core of data analysis consists of, (b) show the necessary steps to carry out this analysis process, (c) review specific techniques for the detection of categories, (d) present examples using the Atlas.ti software, and (e) show the possible ways of presenting the results. Researchers have realized the importance of having methodological works that facilitate the analysis of qualitative data, and allow answering the question: What does qualitative analysis look like in practice?. The development of this type of work pretends on the one hand to facilitate the understanding of the process of qualitative data analysis and, on the other hand, serve to shape better and in a more standard way which was the data analysis procedure applied in the respective investigations. This material should be taken as a first step in the understanding of the process, and it should not be understood that the qualitative analysis is reduced only to what is developed in this article. For example, in the first level grouping step or first coding cycle, the researcher can make use of 25 different types or forms of coding (e.g., live coding). Even so, the development of works such as the present manuscript is intended to facilitate the understanding and reporting the process of qualitative data analysis. Beyond the name with which the researcher calls the analysis procedure carried out, it is relevant to report in his works the basic steps (i.e. Identification, First and Second Level of Categorization), and the specific techniques used to detect categories or topics (e.g. repetition or similarities). Likewise, it is advisable to follow the guidelines recently published by the APA for the publication of qualitative research. We hope that this material will be useful especially for new researchers who need an introductory text to carry out the qualitative data analysis.

Keywords: Qualitative Data Analysis; Basic Core; Techniques to Identify Themes or Categories; Coding; Atlas.ti.

\section{Introducción}

Dentro del proceso de investigación, el análisis de los datos surge como uno de los pasos más importantes (Leech y Onwuegbuzie, 2008). En la investigación cualitativa, el análisis de los datos es una tarea de difícil manejo incluso para los investigadores más experimentados (Basit, 2003; Guest y McLellan, 2003) y la forma de implementar dicha tarea suele acarrear muchas dudas (Souza Minayo, 2012). Por esto, se hace necesario disponer de material que facilite el proceso de análisis.

Aun cuando en la actualidad los investigadores noveles en investigación cualitativa disponen de numerosos manuales que hacen foco en el análisis de datos cualitativos (Gordo López y Serrano Pascual, 2008; Gibbs, 2007; Miles, Huberman y Saldaña, 2014; Saldaña, 2009; Flick, 2014), muchas veces pueden verse confundidos con la gran cantidad de nombres que este tipo de análisis recibe (e.g. Análisis Temático; Análisis de Contenido) o con los diversos métodos cualitativos (e.g. Fenomenología; Teoría fundamentada) que se encuentran disponibles (Cáseres, 2003; 
Escalantes Gomez, 2009; Lawrence y Tar, 2013; Leech y Onwuegbuzie, 2008; Mieles Barrera, Tonon y Alvarado Salgado, 2012; Ryan y Bernard, 2005; Soneira, 2006). Cada uno de estos abordajes cualitativos presenta un lenguaje particular para detallar el proceso de investigación, lo cual dificulta el reconocimiento de los aspectos comunes compartidos por estos métodos (Levitt, et al. 2018). Recientemente, la Asociación Americana de Psicología (APA, Levit, et al. 2018) ha enfatizado la necesidad de identificar, dentro de los diversos métodos y procedimientos cualitativos, estándares compartidos para reportar este tipo de trabajos.

En coincidencia con lo anterior, Dey (1993) y Miles et al. (2014) indicarán que, más allá de la diversidad mencionada, es posible identificar un núcleo básico en lo que respecta al análisis cualitativo sin necesidad de hacer coincidir las distintas perspectivas del método cualitativo (como la Teoría fundamentada, la Etnografía y la Fenomenología). Hacer foco en esta comunalidad les permitirá a los investigadores que se adentren en la investigación cualitativa aproximarse de una manera más sencilla y clara al proceso de análisis de datos (Mayz Díaz, 2008; Rodríguez Sabiote, Lorenzo Quiles y Herrera Torres, 2005).

Como señalan Miles et al. (2014), el proceso de análisis implica fundamentalmente 1) condensación de datos, y 2) presentación de resultados. Siguiendo esta línea, el presente trabajo tiene como objetivos: a) desarrollar en qué consiste el núcleo básico del análisis de datos; b) mostrar los pasos necesarios para llevar a cabo este proceso de análisis; c) revisar técnicas concretas para la detección de categorías; d) presentar ejemplos haciendo uso del software Atlas.ti; y e) mostrar las formas posibles de presentación de los resultados. Investigadores como Basit (2003) han dado cuenta de la importancia de contar con trabajos metodológicos que faciliten el análisis de datos cualitativos y que permitan responder a la pregunta "¿A qué se parece el análisis cualitativo en la práctica?"
(Tuckett, 2005). Siguiendo a Tuckett (2005) y a Levitt et al. (2018), el desarrollo de este tipo de trabajos pretende, por un lado, facilitar la comprensión del proceso de análisis de datos cualitativos $\mathrm{y}$, por otro, servir para que pueda plasmarse mejor, y de una manera más estándar, cuál fue el procedimiento de análisis de datos aplicado en las respectivas investigaciones.

\section{Núcleo básico en el proceso de análisis cualitativo y pasos para llevarlo a cabo}

Más allá de las diferencias entre los abordajes cualitativos aplicados y de las diversas denominaciones con las que se etiquetan las técnicas de análisis, el aspecto fundamental está en cómo categorizar los datos y cómo interconectar estas categorías (Basit, 2003; Guest y McLellan, 2003; Rodríguez Sabiote et al., 2005; Hernández Sampieri, Fernández Collado y Baptista Lucio, 2015). Por categorizar se entiende el proceso mediante el cual el investigador detecta en los datos cualitativos patrones o aspectos de relevancia y los conceptualiza, identificando al mismo tiempo dichos fragmentos con un nombre o etiqueta (i.e. codificándolo). Si bien algunos autores resaltan el hecho de que codificar difiere de categorizar (e.g. Rodríguez Sabiote, et al. 2005), en la práctica suelen superponerse dado que, cuando conceptualizamos parte del material (i.e. categorización), necesariamente tenemos que otorgarle un nombre que identifique dichos fragmentos y los distinga del resto (i.e. codificación). De hecho, el código es el nombre o la etiqueta que ponemos a un segmento de texto para describirlo (Basit, 2003; Miles et al. 2014). En términos metodológicos entonces la codificación es la operacionalización de la categoría o tema, es decir, el nombre con el cual identificamos aquellos fragmentos del dato cualitativo que son relevantes para responder a los objetivos de la investigación, y que pueden agruparse bajo un denominador común (Ryan y Rusell Bernard, 2003; Rodríguez Sabiote, et al. 2005). 
Volviendo al núcleo común, la tarea básica del análisis cualitativo es crear categorías que abarquen tanto aquellas más ligadas a los datos como otras, más abstractas, que agrupan otras categorías. Autores como MacQueen y Milstein (1999) han identificado esta actividad básica en sus propuestas metodológicas, denominándola acto de segmentación. Otros autores, como Westbrook (1994), indican que el análisis contempla trabajar con el dato cualitativo organizándolo, descomponiéndolo en unidades manejables, sintetizándolo y buscando patrones.

Como proponen Ryan y Rusell Bernard (2003), el proceso de análisis se basa fundamentalmente en las siguientes tareas: a) descubrimiento de temas y subtemas; y b) interrelación de los temas. Como se ve, lo fundamental en el análisis está en la detección de patrones, temas o categorías; esto es, en la agrupación de distintos fragmentos bajo un nombre común (Gibbs, 2007; Ryan y Rusell Bernard, 2003).

Por su parte, Hernández Sampieri et al. (2015) mencionan, entre los propósitos centrales del análisis cualitativo: a) explorar los datos e imponerles una estructura (se los organiza en temas o categorías); b) descubrir patrones, temas o categorías en los datos; que a su vez posibiliten c) otorgarles sentido y explicarlos en función del planteamiento del problema. Nuevamente, descubrir temas a partir de los datos recolectados surge como una actividad central en el análisis.

Sintetizando los aportes de los diversos autores, y en base a modelos específicos construidos con esta lógica (e.g. Modelo NCT, Fierse, 2014), puede describirse una serie de pasos necesarios para llevar a cabo el análisis de datos cualitativo antes indicado. Se denominará al primer paso como Identificación. Este paso indica la acción del investigador de identificar, detectar o encontrar en el dato cualitativo (ya sea en formato de texto, imagen, audio, o video) aspectos de interés o relevantes para la investigación. Con el objeto de capturar o hacer patente estos fragmentos, el investigador marcará estas partes de alguna manera. Una pregunta que se puede formular en este punto es: "¿qué aspecto de los datos recolectados es de importancia para responder a los objetivos de la investigación?". Este paso se ha dado también en llamar identificación de unidades de significado (Hernández Sampieri, et al. 2015). La identificación también ha sido ubicada en un paso previo que suele presentarse en el análisis: la trascripción de datos cualitativos a texto. El paso del dato a texto implica ya un proceso de reducción, interpretación y representación (Bailey, 2008).

Por ejemplo, se pone en consideración el siguiente fragmento de respuesta ante la pregunta "¿Qué situaciones académicas les generan emociones negativas y qué hacen para regular dichas emociones?":

Yo por mi parte me pongo muy nerviosa en los exámenes. Para mí es el fin del mundo ir a rendir una materia, no sé por qué, quizás por el hecho de no tener el control total de esa situación y que el resultado no dependa solo de mi desempeño. Eso me deja como en una situación medio vulnerable, creería que es por eso o al menos yo lo percibo así. $Y$ algunas de las cosas que hago frente a eso es respiración, realizo respiración y medito... lo he hecho a veces antes de rendir.

El primer paso consiste en remarcar aquellos fragmentos de las respuestas que sean más significativos para responder a nuestra pregunta de investigación. Luego, en los siguientes pasos, se le dará nombre a esos fragmentos (se los codificará) y se podrán agrupar en temas o categorías más amplias, guiados muchas veces por el conocimiento teórico previo del investigador.

El siguiente paso se puede denominar Agrupación de primer nivel, el cual se refiere a la acción del investigador de detectar patrones o dar significado (i.e. categorizar) a los fragmentos remarcados. Es decir, esos fragmentos pueden ser denominados de determinada manera (i.e. codificados) en base al significado que se le pueda asignar. Este paso se ha denominado en otros trabajos como codifica- 
ción descriptiva (Gibbs, 2007), abierta o de primer nivel (Miles, et al. 2014; Hernández Sampieri, et al. 2015). También se ha denominado este paso como reducción de datos y generación de categorías (Mayz Díaz, 2008;
Rodríguez Sabiote, et al., 2005).

Por ejemplo, siguiendo con el mismo fragmento de respuesta, se pueden identificar en él algunas categorías que pueden ser útiles para la investigación:

"Yo por mi parte me pongo muy nerviosa en los exámenes. Para mí es el fin del mundo ir a rendir una materia, no sé por qué, quizás por el hecho de

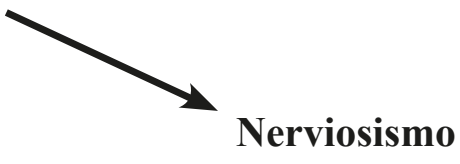

no tener el control total de esa situación y que el resultado no dependa solo de mi desempeño. Eso me deja como en una situación medio vulnerable, creería que es por eso o al menos yo lo percibo así. $\mathrm{Y}$ algunas de las cosas que hago frente a eso es respiración, realizo respiración y medito... lo he

\section{Respiración}

hecho a veces antes de rendir."

El paso siguiente puede denominarse como de Agrupación de segundo nivel, en el que se pasa de una categorización más ligada a los datos, dado que se basa en fragmentos específicos del texto, a una categorización de mayor nivel de abstracción o conceptualización, dado que se empiezan a conceptualizar las mismas categorías iniciales. En este momento, se empiezan a interconectar también las distintas categorías (Friese, 2014). Otros autores han descripto este proceso como el paso de una codificación descriptiva a otra más analítica (Gibbs, 2007), el paso de una codificación abierta de primer nivel a una axial de segundo nivel (Miles, et al. 2014; Hernández Sampieri, et al. 2015), o el paso desde un primero a un segundo ciclo de codificación (Saldaña, 2009). Dentro del contexto de la Teoría fundamentada se habla del paso de una codificación abierta, a otras de tipo axial y selectiva, y se hace mención al método de comparación constante (Lawrence y Tar, 2013; Soneira, 2006).

A manera de ejemplo, a continuación se muestra una agrupación de mayor nivel de abstracción. Como puede verse, el nerviosismo puede conceptualizarse o integrarse a la categoría de emociones negativas, mientras que la respiración conforma un tipo de regulación emocional que se enfoca en la respuesta afectiva. Asimismo, se procedió a ligar ambas categorías para dar cuenta de la relación que mantienen entre sí. En el caso del ejemplo, lo que se muestra es que ante emociones negativas generadas en el contexto académico, una de las formas en que los estudiantes regulan dicha emoción (ansiedad o nerviosismo) es actuando sobre la propia respuesta emocional intentando disminuirla, por ejemplo por medio de la respiración o la meditación: 
"Yo por mi parte me pongo muy $\longrightarrow$ Nerviosismo $\longrightarrow$ Es parte de

nerviosa en los exámenes. Para mí

es el fin del mundo ir a rendir una

materia, no sé por qué, quizás por el

hecho de no tener el control total de

esa situación y que el resultado no

dependa solo de mi desempeño. Eso

me deja como en una situación medio

vulnerable, creería que es por eso o

Emociones negativas

al menos yo lo percibo así. Y algunas

de las cosas que hago frente a eso

es respiración, realizo respiración y

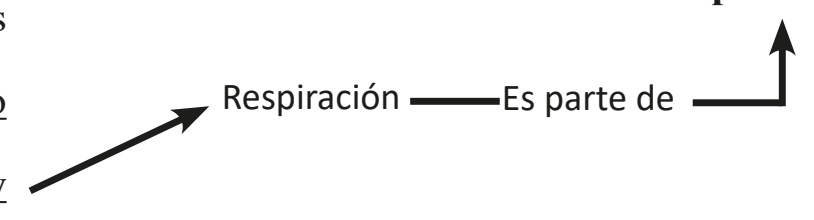

Regulación enfocada en la respuesta emocional

medito... lo he hecho a veces antes de

rendir"

Hasta acá se indicó que la tarea básica de aquel que se adentra en el análisis cualitativo consiste en categorizar los datos, en otras palabras, darles significado y etiquetarlos. Para lograr esto con mayor facilidad pueden definirse una serie de pasos, tres concretamente, que van desde la identificación a la generación de categorías de distinto nivel de abstracción. Ya que el proceso de generación de categorías es el punto central, a continuación se verán algunas técnicas que facilitan dicha categorización.

\section{Técnicas para la identificación de temas o categorías}

Como se mencionó, el paso central del análisis cualitativo consiste en la identificación de temas o categorías. Recientemente Ryan y Rusell Bernard (2003) presentaron unas serie de técnicas que el investigador puede aplicar para facilitar la identificación en los datos de estos temas y categorías. Los autores comienzan preguntándose “Cómo nos damos cuenta cuando estamos frente a un tema o una categoría?". Uno se da cuenta, según Ryan y Rusell Bernard (2003), cuando puede responder a la siguiente pregunta: “¿Esta expresión es un ejemplo de qué?”. En otras palabras, a qué se está refiriendo esta expresión. Esa es entonces la pregunta básica que hay que hacer para comenzar a identificar categorías en nuestros datos. Siguiendo con el ejemplo, al realizar esta pregunta podemos decir que el nerviosismo es un ejemplo de una emoción negativa, y que la respiración forma parte de un tipo específico de regulación emocional.

Otra técnica que se menciona es prestar atención a las repeticiones, es decir, a temáticas que aparecen recurrentemente, o de manera frecuente, en los datos que se analizan. Los autores dicen que "mientras más aparezca un concepto en el texto, más probablemente se tratará de un tema" (Ryan y Russell Bernard, 2003, p. 89). Así, en este caso, es el mismo 
concepto el que se repite a lo largo de los datos analizados (i.e. un tema muy frecuente). Veamos los distintos fragmentos que se toman a continuación:

"Yo, por mi parte, me pongo muy nerviosa en los exámenes...".

"Cuando tengo que rendir o pensar en el examen automáticamente empiezo a traspirar...".

“...sí, cuando tenemos exámenes los días previos me siento muy intranquilo y con ganas de vomitar...".

Es posible ver en las distintas respuestas que se está haciendo referencia, aunque con matices particulares, a un mismo tema o categoría, que tiene que ver con el nerviosismo que genera la situación de examen.

Se menciona además la técnica de buscar similitudes y diferencias. Mediante la comparación sistemática de las unidades de análisis (e.g. fragmentos de texto o audio) se intenta responder a la siguiente pregunta: “¿De qué manera es similar o diferente este fragmento con respecto al fragmento precedente o procedente?". Por ejemplo, veamos la siguiente respuesta:

“A mí, en lo particular, los exámenes no me generan esa ansiedad... yo me siento tranquilo porque pienso ¿qué es lo peor que me puede pasar?".

$\mathrm{Si}$ se lo compara con las anteriores respuestas, que referían claramente a un estado ansioso o de nerviosismo, en este caso se genera una emoción positiva de tranquilidad, en base a otra estrategia diferente de regulación emocional como puede ser la puesta en perspectiva de la situación.

También es posible identificar material relacionado teóricamente, es decir, poder responder cómo el dato cualitativo da cuenta de categorías definidas teóricamente. Básicamente, esta última técnica permitiría trabajar de un modo más deductivo, esto es, partir de definiciones o conceptos teóricos (i.e. categorías previas) prestando atención a su aparición en los datos. En los ejemplos anteriores es posible apreciar que se han usado conceptos tales como regulación enfocada en la respuesta emocional para la generación de las categorías. Estas estrategias mencionadas de regulación emocional forman parte de algunos modelos teóricos que trabajan estos procesos (e.g. Gross, 2015). La técnica que aquí se menciona refiere a que el investigador conoce estos conceptos previamente e intenta ver en qué medida se presentan en la respuestas de los participantes.

Sintetizando las técnicas anteriormente mencionadas y tomando los criterios propuestos por Miles y Huberman (1994), básicamente se dispone de dos grandes métodos para la creación de códigos o categorías: a) el método inductivo (que incluye principalmente las técnicas de repeticiones; similitudes y diferencias); y b) el método deductivo (que incluye identificar material relacionado teóricamente).

A continuación se mostrará cómo se pueden aplicar los pasos del análisis de datos cualitativos haciendo uso del software Atlas. ti, software que facilita también la aplicación de las técnicas de detección de categorías anteriormente desarrolladas.

\section{Pasos para el análisis de datos cualitativos mediante Atlas.ti.}

En primer lugar, es importante considerar los distintos softwares especializados como herramientas de análisis de datos cualitativos, o, dicho en otras palabras, como herramientas informáticas que brindan facilidades de análisis (e.g. herramientas de búsqueda de texto, facilidades de codificación, facilidades en la escritura de memos o anotaciones, facilidades en la diagramación de relaciones entre las categorías surgidas del análisis). Esto posibilita una mayor sistematización y transparencia en el análisis, así como una merma de los posibles errores en los que puede incurrir el investigador al tener que analizar grandes cantidades de datos (Atkisson, Monaghan y Brent, 2010; Chernobilsky, 2006; Guest et al. 2012). Dicho esto, el lector debe quedar 
advertido que el software de análisis de datos cualitativos que utilice no interpretará por sí mismo los datos, ni llevará a cabo acciones que son propias del investigador, como encontrar en los datos ciertos patrones comunes que lleven a pensar en algún tipo de relación. Por el contrario, estas y muchas otras competencias deben formar parte del repertorio del investigador y no pueden ser suplantadas por el software (Basit, 2003; Rodríguez Sabiote, et al. 2005). En suma, como señala Chernobi1sky (2006), el programa de análisis no podrá analizar los datos en lugar del investigador.

En segundo lugar, la elección del software va estar muy determinada por los objetivos propios de la investigación y por el tipo de análisis que el investigador intente aplicar a los datos (Basit, 2003). En este caso concreto, se mostrará el uso que puede hacerse de Atlas. ti y la forma de aplicar los pasos definidos para el análisis de datos. Para todos los ejemplos se utilizó la versión 7.5.4 del software. Además, se mostrará cómo puede el investigador directamente trabajar con un audio sin necesidad de hacer una transcripción previa de sus entrevistas. Esto se hace con el fin de mostrar la versatilidad del software, que además permite trabajar con otros formatos como video o imágenes (Flores-Kanter, 2014).

\section{Paso 1. Identificación de unidades de} significado.

Con la opción de "crear cita", Atlas.ti permite ir subrayando o remarcando aquellos fragmentos del dato (en este caso, un audio) que sean de relevancia para responder a los objetivos de investigación.

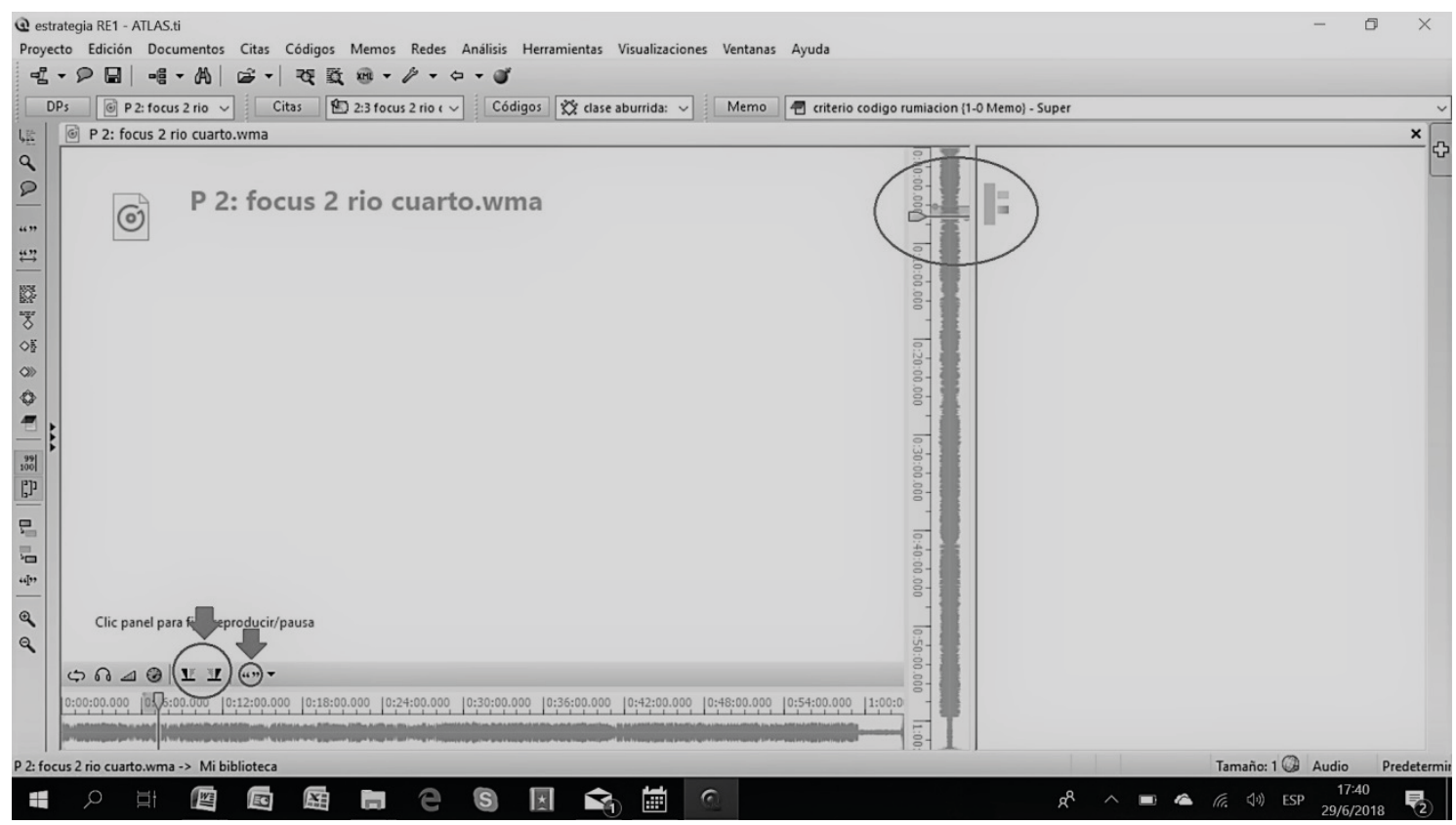

Figura 1. Identificación de unidades de significado con Atlas.ti.

En el caso de la Figura 1 se está marcando un fragmento de audio, pero es lo mismo si uno trabajara directamente con un texto. En este ejemplo se cita y remarca la siguiente respuesta:

"Yo, por mi parte, me pongo muy nerviosa en los exámenes. Para mí es el fin del mundo ir a rendir una materia, no sé por qué, quizás por el hecho de no tener el control total de esa situación y que el resultado no dependa solo de mi desempeño. Eso me deja como en una situación medio vulnerable, creería que es por eso o al menos yo 
lo percibo así. Y algunas de las cosas que hago frente a eso es respiración, realizo respiración y medito... lo he hecho a veces antes de rendir"

Paso 2. Agrupación de primer nivel: codificación o creación de categorías.

Para proceder a la etapa de codificación, Atlas.ti ofrece la opción de crear y asignar nuevos códigos:

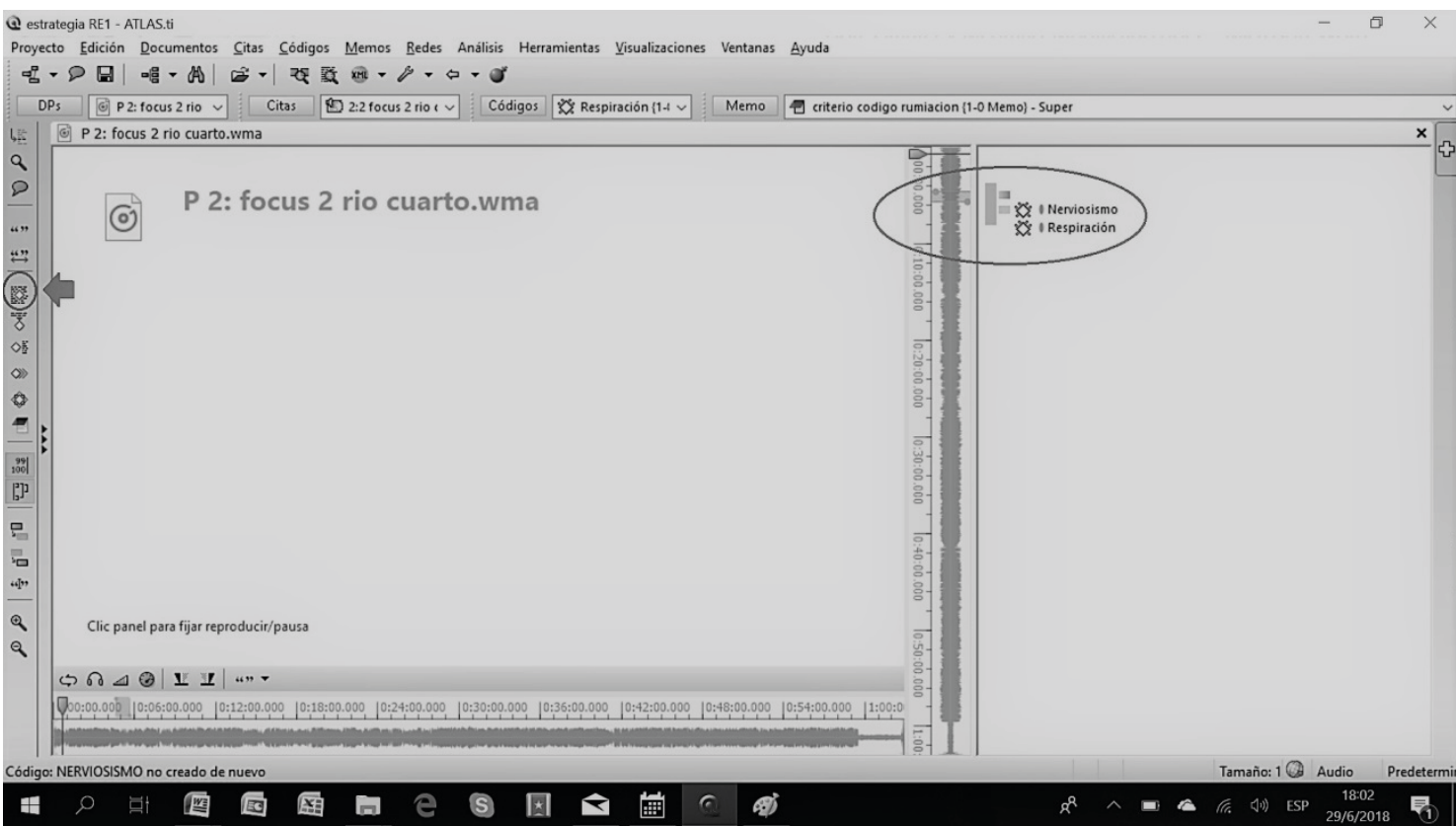

Figura 2. Codificación de primer nivel en Atlas.ti.

La Figura 2 muestra que en la sección remarcada se pueden identificar dos categorías (códigos en Atlas.ti) identificadas como Nerviosismo y Respiración.

Paso 3. Agrupación de segundo nivel: creación de categorías mayor nivel de abstracción e interrelación de las categorías.

Este paso es más sencillo de llevar a cabo con la opción de Redes. Dentro del panel para diagramar las redes, es posible indicar cómo deben quedar subsumidas las distintas categorías creadas en los pasos previos. También posibilita crear nuevas categorías. Esto es especialmente importante dado que, dentro del panel de redes, se pueden ir agrupando las categorías iniciales en nuevas categorías con mayor nivel de abstracción. 


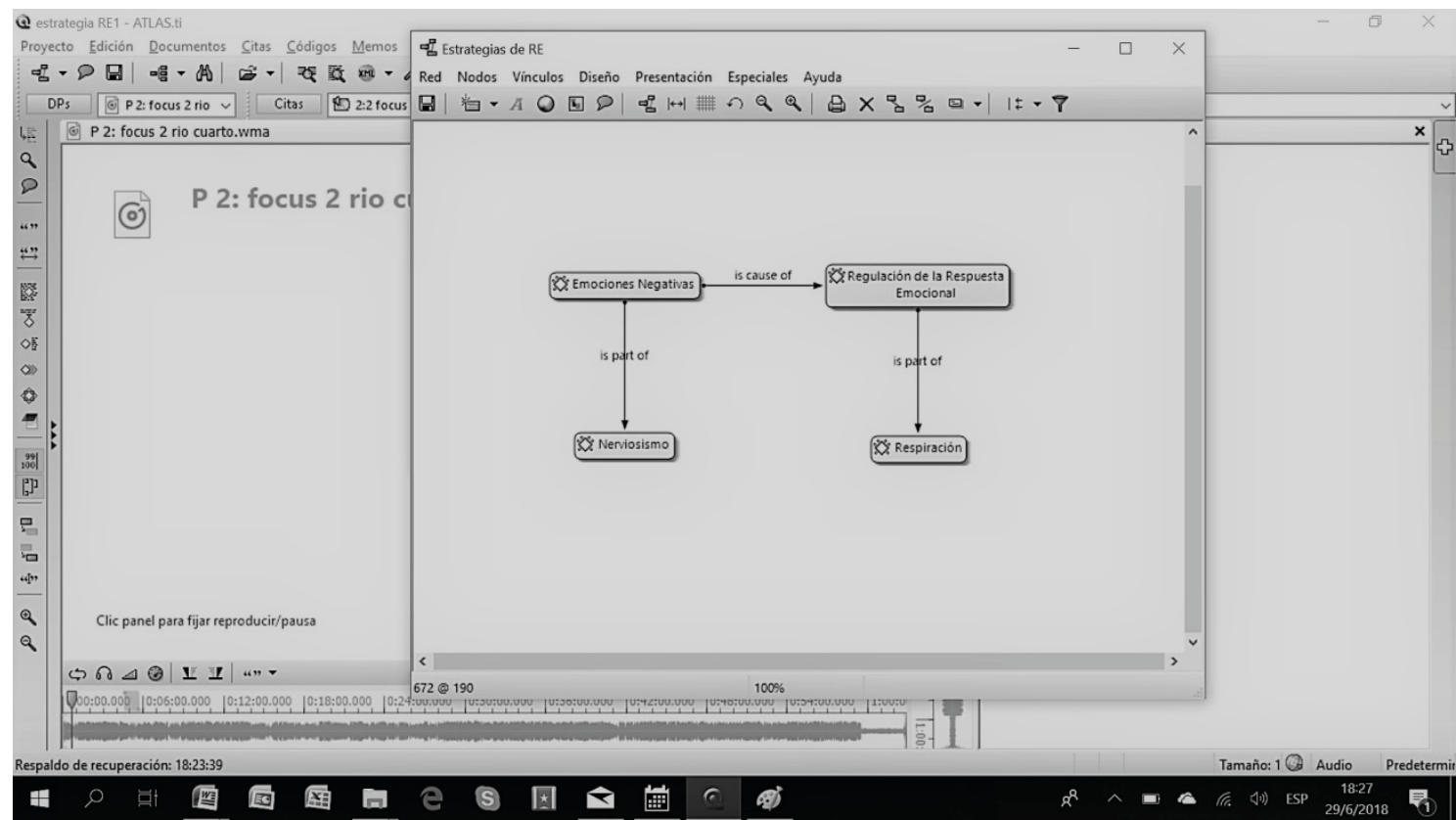

Figura 3. Interrelación y agrupación de códigos en Atlas.ti.

Como puede verse, el nerviosismo puede conceptualizarse o integrarse a la categoría de emociones negativas, mientras que la respiración conforma un tipo de regulación emocional que se enfoca en la respuesta afectiva. El software también permite ligar ambas categorías dando cuenta de la relación que mantienen entre sí. Por supuesto, a medida que el investigador avanza en el análisis, esta red se irá complejizando, integrando e interrelacionando con un número mayor de categorías.

\section{Formas de presentación de los resultados del análisis cualitativo}

Finalmente, se abordarán las formas posibles que tiene el investigador de dar a conocer los resultados obtenido del análisis de datos cualitativos. Existen tres formas básicas de presentación de resultados. Algunas de estas se señalan en la literatura especializada (Guest, et al. 2012; Miles, et al. 2014) y otras pueden identificarse en los trabajos que llevan a cabo este tipo de análisis cualitativo. Entre las principales formas de presentación de los resultados se puede identificar: a) la narrativa, b) mediante tablas, y c) mediante gráficas. Es importante mencionar que todas estas formas de presentación pueden mostrarse de modo conjunto o combinado y no es necesario elegir entre una u otra.

Unas de las formas más comunes de presentación es la narrativa, (e.g. Giaconi Mores, Pedrero Sanhueza y San Martín Peñailillo, 2017). En este modo de presentación de resultados, es importante que el investigador describa adecuadamente cuáles fueron las categorías o temáticas que surgieron del análisis y que sustente estas categorías con respuestas textuales tomadas de los propios participantes.

Siguiendo con el ejemplo de las emociones negativas ante situaciones académicas, se podría realizar la siguiente presentación narrativa:

Unas de las situaciones académicas que más generan emociones negativas son las situaciones de examen. Ante estas situaciones los alumnos suelen experimentar emociones negativas como el nerviosismo: "Yo por mi parte me pongo muy nerviosa en los 
exámenes. Para mí es el fin del mundo ir a rendir una materia".

Otra forma de presentar los resultados es haciendo uso de tablas, tal como se muestra en el trabajo de Gallardo Echenique, Marqués Molías y Bullen (2015). Mediante una matriz, se puede ir mostrando en los encabezados de las filas y las columnas cuáles son las categorías o temáticas surgidas del análisis, mientras que en los cruces de las filas y columnas se pueden colocar las respuestas textuales de los participantes.

Siguiendo con el mismo ejemplo, se describen los resultados en la tabla 1:

Tabla 1. Situaciones académicas que generan emociones negativas.

Emociones negativas

Nerviosismo

Finalmente los resultados también pueden mostrarse de manera gráfica o mediante redes (e.g. Trógolo, Flores-Kanter y Medrano, 2014). Esto es justamente lo que representa la Figura 3, en la que se ve que el nerviosismo se integra a la categoría de emociones negativas, mientras que la respiración conforma un tipo de regulación emocional que se enfoca en la respuesta afectiva.

\section{Conclusiones finales}

El análisis cualitativo constituye un proceso de suma complejidad, que demanda mucha experiencia e idoneidad por parte de quienes lo producen. Este trabajo se propuso como principal objetivo ofrecer una síntesis de los aspectos centrales del análisis cualitativo para que, a partir de su lectura, los investigadores no experimentados en estos métodos puedan introducirse en el complejo proceso del análisis cualitativo. Sin embargo, es importante remarcar que el núcleo básico que se ha desarrollado en este artículo es válido para aquellos abordajes que requieren reducir la información cualitativa a categorías o códigos (e.g. Teoría fundamentada; Análisis de contenido), que se ubican dentro de los métodos de análisis de tradición sociológica y que,
Exámenes

"Yo por mi parte me
pongo muy nerviosa en
los exámenes. Para mí
es el fin del mundo ir a
rendir una materia"

además, trabajan con textos o narrativas obtenidos libremente, como discursos o respuestas a preguntas abiertas (Ryan y Bernard, 2005). El lector interesado en otras formas de análisis cualitativo puede consultar el compendio de las principales técnicas disponibles llevado a cabo por Leech y Onwuegbuzie (2008). En este sentido, debe tomarse este material como un primer paso en la comprensión del proceso, y no debe entenderse que el análisis cualitativo se reduce únicamente a lo desarrollado en este artículo. Por ejemplo, en el paso de agrupación de primer nivel o primer ciclo de codificación, el investigador puede hacer uso de 25 formas o tipos distintos de codificación (e.g. codificación en vivo; ver Saldaña, 2009). Aun así, el desarrollo de trabajos como el presente pretenden facilitar la comprensión y el proceso de análisis de datos cualitativos de los investigadores que se adentran en este tipo de metodología, y, además, servir para que pueda dejarse mejor plasmado cuál fue el procedimiento de análisis de datos aplicado en las respectivas investigaciones (Levitt, et al. 2018; Tuckett, 2005). Más allá del nombre con el cual el investigador denomine el procedimiento de análisis efectuado, es relevante que reporte en sus trabajos los pasos básicos (i.e. Identificación; Categorización de primer $\mathrm{y}$ 
segundo nivel) y las técnicas específicas utilizadas para la detección de categorías o temas (e.g. repetición o similitudes). Asimismo, se aconseja seguir los lineamientos recientemente publicados por la APA (Levitt, et al. 2018) para la publicación de investigaciones cualitativas. Esperamos que este material sea de utilidad especialmente para los investigadores noveles que necesitan de un texto introductorio para llevar a cabo el análisis de datos cualitativo.

\section{Referencias bibliográficas}

Atkisson, C., Monaghan, C. y Brent, E. (2010). Using computational techniques to fill the gap between qualitative data analysis and text analytics. KWALON, 15(3), 6-19.

Bailey, J. (2008). First steps in qualitative data analysis: transcribing. Family Practice, 25, 127-131. Doi: 10.1093/fampra/cmn003

Basit, T. (2003). Manual or electronic? The role of coding in qualitative data analysis. Educational Research, 45(2), 143-154. doi: 10.1080/0013188032000133548

Cáseres, P. (2003). Análisis cualitativo de contenido: Una alternativa metodológica alcanzable. Psicoperspectivas, 2(1), 53-81. Doi: 10.5027/ psicoperspectivas-Vol2-Issue1-fulltext-3

Chernobilsky, L. B. (2006). El uso de la computadora como auxiliar en el análisis de datos cualitativos. En Gialdino, I. V. Estrategias de investigación cualitativa (pp. 239-271). Barcelona: Gedisa Editorial.

Dey, I. (1993). Qualitative data analysis. A userfriendly guide for social scientists. London: Taylor, \& Francis Group.

Escalantes Gomez, E. (2009). Perspectivas en el análisis cualitativo. Theoria, 18(2), 55-67.

Flick, U. (2014). The Sage Handbook of Qualitative Data Analysis. Los Angeles: Sage Publication.

Flores-Kanter, P. E. (2014). Análisis de Videos mediante Atlas.ti: Ejemplo de un Estudio sobre Apelaciones Emocionales en Spots Electorales Televisivos. Revista de Ciencia y Técnica. Universidad Siglo 21.
Friese, S. (2014). Qualitative Date Analysis with ATLAS.ti. EE.UU, Los Angeles: SAGE publications.

Gallardo Echenique, E., Marqués Molías, L. y Bullen, M. (2015). El estudiante en la educación superior: Usos académicos y sociales de la tecnología digital. RUSC. Universities and Knowledge Society Journal, 12(1), 25-37. doi: 10.7238/rusc.v12i1.2078

Giaconi Mores, C., Pedrero Sanhueza, Z. y San Martín Peñailillo, P. (2017). La discapacidad: Percepciones de cuidadores de niños, niñas y jóvenes en situación de discapacidad. Psicoperspectivas, 16(1), 55-66. doi: 10.5027/psicoperspectivas-vol16-issue1-fulltext-822

Gibbs, G. R. (2007). Análisis de Datos Cualitativos. Madrid: Morata.

Gordo López, A. J. y Serrano Pascual, A. (2008). Estrategias y Prácticas Cualitativas de Investigación Social. Madrid: Pearson. Prentice Hall.

Guest, G., MacQueen, K. M. y Namey, E. E. (2012). Applied Thematic Analyisis. EE.UU, Los Angeles: SAGE publications.

Guest, G. y McLellan, E. (2003). Distinguishing the trees from the forest: Applying cluster analysis to thematic qualitative data. Field Methods, 15(2), 186-201. doi: 10.1177/1525822X03251188

Gross, J. J. (2015). Emotion Regulation: Current Status and Future Prospects. Psychological Inquiry, 26(1), 1-26. doi:10.1080/1047 840x.2014.940781

Hernández Sampieri, R., Fernández Collado, C. y Baptista Lucio, P. (2015). Metodología de la Investigación. Madrid: Mc Graw Hill.

Lawrence, J. y Tar, U. (2013). The use of Grounded Theory Technique as a practical tool for qualitative data collection and analysis. The Electronic Journal of Business Research Methods, 11(1), 29-40.

Leech, N. L. y Onwuegbuzie, A. J. (2008). Qualitative data analysis: A compendium of techniques and a framework for selection for school psychology research and beyond. School Psychology Quarterly, 23(4), 587-604. doi: 10.1037/1045-3830.23.4.587 
Levitt, H. M., Creswell, J. W., Josselson, R., Bamberg, M., Frost, D. M., Suárez-Orozco, C. (2018). Journal article reporting standards for qualitative primary, qualitative meta-analytic, and mixed methods research in psychology: The APA publications and communications board task force report. American Psychologist, 73(1), 26-46. doi: 10.1037/amp0000151

MacQueen, K. M. y Milstein, B. (1999). A systems approach to qualitative data management and analysis. Field Methods, 11(1), 27-39.

Mayz Díaz, C. (2008). ¿Cómo desarrollar, de una manera comprensiva, el análisis cualitativo de los datos? Educere, 13(44), 55-66.

Mieles Barrera, M. D., Tonon, G. y Alvarado Salgado, S. V. (2012). Investigación cualitativa: El análisis temático para el tratamiento de la información desde el enfoque de la fenomenología social. Universitas Humanística, 74, 195-225.

Miles, M. B. y Huberman, A. M. (1994). Qualitative Data Analysis: An Expanded Sourcebook. Thousand Oaks, California: Sage Publication.

Miles, M. B., Huberman, M. A. y Saldaña, J. (2014). Qualitative Data Analysis. A Methods Sourcebook. Los Angeles: Sage Publication.

Rodríguez Sabiote, C., Lorenzo Quiles, O. y Herrera Torres, L. (2005). Teoría y práctica del análisis de datos cualitativos. Proceso general y criterios de calidad. Revista Internacional de Ciencias Sociales y Humanidades, 15(2), 133-154.
Ryan, G. W. y Russell Bernard, H. (2003). Techniques to identify themes. Field Methods, 15 (1), 85-109. doi: 10.1177/1525822X02239569

Ryan, G. W. y Russell Bernard, H. (2005). Data Management and Analysis Methods. En Denzin, N. K. y Lincoln, Y. S., Handbook of Qualitative Research. London: Sage Publication.

Saldaña, J. (2009). The Coding Manual for Qualitative Researchers. EEUU: Sage Publication.

Soneira, J. A. (2006). La "Teoría fundamentada en los datos" (Grounded Theory) de Glaser y Strauss. En Vasilachis de Gialdino, I. Estrategias de investigación cualitativa. Barcelona: Editorial Gedisa.

Souza Minayo, M. C. (2012). Análise qualitativa: teoria, passos e fidedignidade. Ciênc. saúde coletiva, 17(3), 621-626. doi: 10.1590/S141381232012000300007

Trógolo, M. A., Flores Kanter, P. E. y Medrano, L. A. (2014). Análisis de Estrategias de regulación emocional en estudiantes universitarios de la ciudad de córdoba. En Cardozo, G. (ed.), El psicólogo del tercer milenio: nuevas realidades, nuevos desafios (pp. 31-45). Córdoba: EDUCC.

Tuckett, A. G. (2005). Applying thematic analysis theory to practice: A researcher's experience. Contemporary Nurse, 19(1-2), 75-87. doi: 10.5172/conu.19.1-2.75

Westbrook, L. (1994). Qualitative research methods: A review of Major stages, data analysis techniques, and quality controls. LISR, $16,241-254$.

Recibido: 18 de diciembre de 2017 Aceptado: 29 de octubre de 2019 
